\title{
The motion and shape of a bubble in highly viscous liquid flowing through an orifice
}

\author{
C.-H. Chen, B. Hallmark, J. F. Davidson. \\ University of Cambridge Department of Chemical Engineering and Biotechnology, Philippa Fawcett \\ Drive, Cambridge, CB3 0AS.
}

\begin{abstract}
Experiments and theory concern the behaviour of a small bubble carried through an orifice by a very viscous liquid. The liquid was polybutene oil, of viscosity about $70 \mathrm{~Pa} \mathrm{~s}$, i.e. 70,000 times that of water. The Reynolds number of the flow is substantially less than one, hence the flow pattern is approximately radial flowing into, and away from, the orifice. These flow patterns have profound effects on the shape of an entrained bubble. On the upstream side, the acceleration of the liquid, as it approaches the orifice, causes elongation of the bubble since the front of the bubble moves faster than the back. On the downstream side, the reverse occurs: the back of the bubble moves fast than the front. Thus the height of the bubble diminishes as it moves away from the orifice, leading to the formation of a 'crescentmoon' shape. The shape of these bubbles can be predicted by considering the motion of a droplet of the same liquid replacing the bubble: the resulting geometric theory gives good predictions of bubble deformation approaching the orifice and of 'crescent-moon' formation downstream of the orifice.
\end{abstract}

Keywords: Two-phase flow, bubble deformation, geometric theory, viscous flows, Multipass rheometer

(c) CHC, BH, JFD. May $8^{\text {th }} 2019$ - Revision 1

Submitted to Chemical Engineering Science for consideration. 


\section{Introduction and literature survey}

Highly viscous liquids containing gas bubbles are used to make foams. It therefore seems appropriate to study the behaviour of a single bubble in a very viscous liquid, flowing through an orifice, to answer questions as follows. How does the bubble deform on passing through the orifice? Does the bubble change shape? Does it break up and, if so, how?

These questions were answered by using a multipass rheometer (MPR) (Mackley et al., 1995): the MPR contains two pistons and cylinders, the pistons moving to-and-fro synchronously, so as to induce toand-fro motion of liquid through an orifice connected to the two cylinders. The orifice was constructed of stainless steel, but held in place by quartz glass windows such that bubbles in the liquid could be observed as they deformed. The liquid used was a polybutene viscosity standard (Brookfield): a Newtonian fluid with a viscosity of $68 \mathrm{~Pa}$. This liquid was chosen to explore bubble deformation in capillary number regimes that cannot be obtained with lower viscosity media, such as water, and is representative of the viscosity of some polymer foams at high shear rate (Wang, 2009).

The motion and behaviour of single bubbles was observed by high speed photography. Remarkable changes of bubble shape were observed when a single bubble passed through an orifice:

i. as a bubble approached the orifice, the bubble elongated in the direction of flow;

ii. after a bubble had passed through the orifice, the liquid motion on the downstream side caused the bubble to take on the shape of a crescent moon;

iii. rather than undergoing break-up, the crescent-shaped bubble returned to its initially spherical shape significantly downstream of the orifice.

A simple kinematic theory is presented in this paper that is able to give insight into these experimental observations: it assumes that the bubble deformation is the same as that of a 'marked drop' of liquid embedded within the parent liquid. The key assumption underlying this theory is that the bubbles are effectively 'passive markers' embedded within the liquid phase, corresponding to a capillary number regime where viscous effects are dominant.

Fluids consisting of gas-liquid mixtures are widespread in both nature and industry. Bubbly liquids, typically containing around $50 \%$ by volume of liquid (Torres et al., 2015), are encountered in volcanic magmas (Mader et al., 2013), in foamed cements (Amran et al., 2015) and in the food industry (Mezzenga et al., 2005). Foams are classified either as 'wet', with liquid content ranging between 15\% and $36 \%$ on a volumetric basis, or dry, where the liquid content is typically less than $5 \%$ (Drenckhan and Hutzler, 2015). Uses of foam are widespread in engineering (Eaves, 2004), the food industry (Narsimhan and Xiang, 2018) and are commonly encountered in the natural world (Hill and Eastoe, 2017). 
Often, bubbly liquids or wet foams are precursors to the formation of porous solids. The gas bubbles become voids within a matrix of solidified liquid and serve to enhance the properties of the resultant material. For example: foamed concrete has a lower density than its non-voided counterpart (Amran et al., 2015), making it more suitable for cementing oil wells in fragile rock structures, and foamed polyurethane has a thermal conductivity an order of magnitude lower than that of the single phase polymer (Collishaw and Evans, 1994). The ultimate properties of the porous solid are dependent on those of the single-phase materials and on the structure that results from the distribution of voids within the solid.

The behaviour of gas bubbles contained within a flowing liquid is, therefore, of relevance to the design of 'engineered' porous solids. This has been the subject of research spanning many decades: phenomena such as Ostwald ripening (Ostwald, 1897; Voorhees, 1985), bubble breakup and bubble coalescence (Prince and Blanch, 1990) all affect the structure and distribution of the gas phase. Moreover, when bubbles rise through a liquid due to buoyancy there are fundamental differences between the dynamics of a single bubble (Wallis, 1974) and swarms of bubbles (Richardson and Zaki, 1954). It is within the context of this background that we wish to address the questions posed in the introduction.

As the viscosity of the liquid phase increases, and as the effect of inertia diminishes, bubble shape is governed primarily by the ratio of viscous deformation forces to surface tension forces, described by the capillary number, $\mathrm{Ca}$. Of particular relevance to the work presented here are theoretical and experimental studies concerning the deformation of bubbles as they flow through ducts and constrictions at low Reynolds number and with 'moderate' to 'high' capillary number.

Theoretical studies of the deformation of bubbles in drops in various flow fields have been wellsummarised in the literature (Rallison, 1984). Studies involving simple shear flows are of relevance: slender body theory has been developed to describe the deformation of droplets as $\mathrm{Ca} \rightarrow \infty$ (Hinch and Acrivos, 1980), with Rallison providing theory to describe droplet deformation at arbitrary capillary number (Rallison, 1984). This latter study concluded that low viscosity drops (i.e. bubbles) can attain highly stable, elongated, shapes: these shapes may have pointed tips, or cusps. Extensive theoretical investigation of the formation and stability of cusped interfaces has subsequently been carried out (Pozrikidis, 1998; Shikhmurzaev, 1998; Siegel, 2000).

Long, slender bubbles having cusped tips have been observed experimentally in simple shear flows over the range $0.01<C a<10$ (Rust and Manga, 2002): these observations were in good agreement with theory. Further research by the same authors identified the presence of similarly-shaped bubbles in a solidified magma (Rust et al., 2003). Aerofoil-shaped bubbles, with just a single cusp, have been observed in pipe flow for $\mathrm{Ca}>1$ (Kameda et al., 2008). The shape of these bubbles departed from the twin-cusp slender bodies observed in simple shear flow due to the fully three-dimensional Poiseuille nature of the pipe flow: theory developed for two-dimensional Poiseuille flow (Richardson, 1973) was 
able to capture some of the observed features. Observations of bubbles flowing through a Hele-Shaw cell, where $6 \times 10^{-5}<C a<2 \times 10^{-2}$, revealed a rich variety of shapes such as elongated bubbles, 'short-tail' and 'long-tail' bubbles (Kopf-Sill and Homsy, 1988): the broad spectrum of bubble shapes was attributed to the fully three-dimensional nature of the flow field.

The flow-field produced as liquid flows through a constriction or an orifice is substantially more complex than that for a simple shear flow or a Poiseuille flow: the flow-field will contain extensional and shear components and the capillary number will vary as a function of location. Measurements of deformation and velocity have been made for a bubble contained with a flow of $1.05 \mathrm{~Pa}$ s silicone oil as it passes through a narrow orifice (Dawson et al., 2015). Bubble elongation upstream of the orifice was observed, with bubbles taking mildly concave shapes downstream of the orifice. Under some circumstances, cusp formation was observed as the bubble passed through the orifice.

A theoretical investigation of the behaviour of liquid droplets suspended in a continuous liquid phase flowing through a rounded constriction has been done by Khayat and co-workers (Khayat et al., 2000). For the case where the viscosity ratio between the continuous and discrete phases is 4 , they compute the formation of highly deformed 'crescent-moon' droplets if an initially spherical droplet is placed in the centre of the constriction prior to the commencement of flow. Zero surface tension was assumed in this study, hence $\mathrm{Ca}=\infty$. Shapes similar to those observed by Dawson were also computed for an initially spherical droplet placed upstream of the constriction prior to the commencement of flow. Experimental work has recently been undertaken (Sauzade and Cubaud, 2018) that examines the deformation of bubbles as they pass through a series of rounded constrictions, similar to those used in the theoretical study of Khayat and co-workers. Crescent-moon shaped deformation was not observed in this geometry, probably due to the nature of the periodically constricting channel, but a number of differently-shaped bubbles containing cusps were observed.

\section{Experimental}

\subsection{Apparatus}

Figure 1(A) shows the essential features of the apparatus. The viscous liquid is shown in white. The orifice plate is shown in black within the black, dashed, square. Two pistons, indicated in black, go back and forth synchronously, pushing the liquid to-and-fro through the orifice plate.

\section{$<$ FIGURE 1>}

The apparatus is quasi 'two-dimensional': the orifice and the region marked in white contained in the plane of the diagram are sandwiched between two glass windows. These windows, separated by a distance of $1 \mathrm{~mm}$ normal to the plane of the diagram, set the thickness of the flow channel. The pistons, black in Figure 1(A) are cylindrical, of diameter $10 \mathrm{~mm}$, with a connecting piece to transfer the liquid from the cylinders to the observation region. The width of the channel immediately before and after the 
orifice is the same as the piston diameter, i.e. $10 \mathrm{~mm}$. The shape of the flow constriction results from two stainless steel inserts, which are sandwiched between the glass windows. These inserts are interchangeable and can take the form of an orifice, shown, or contraction-expansion channels with various contraction angles. The orifice plate that is illustrated in Figure 1(B) has an opening $1 \mathrm{~mm}$ wide and plate thickness of $1 \mathrm{~mm}$. The light source and camera, shown in Figure 1(A), were positioned to observe the flow constriction such that the axis joining the light source and camera was normal to the plane of the diagram.

A high-speed CMOS camera (Basler acA1300-200HC) was used with an LED light source (Thorlabs MCWWHCP1) to obtain the bubble images. The test liquid was a Newtonian polybutene oil (Brookfield viscosity standard B7300, 73.0 Pa s at $25^{\circ} \mathrm{C}$ ). A rotational rheometer (TA Instruments ARES) was used to verify the viscosity of the test liquid: the results, which are available in the supplementary material ${ }^{1}$, demonstrated that the liquid was Newtonian with an apparent viscosity of $67 \mathrm{~Pa}$ s. The difference from the 73.0 $\mathrm{Pa}$ s quoted above is probably due to temperature effects. The surface tension of was assumed to be $30 \mathrm{mN} / \mathrm{m}$, an estimate based on polybutene oils of similar viscosity (Lewandowski and Dupuis, 1994).

\subsection{Experimental procedure}

Polybutene oil was first loaded into the test chamber of the MPR, shown in white in Figure 1(A). Air bubbles were then injected into the chamber using a syringe with a hypodermic needle. These bubbles were stably suspended in the liquid: the effects of buoyancy and of Ostwald ripening were slow due to the liquid's high viscosity. The flow chamber was formed by selecting different quartz glass windows and stainless steel inserts to obtain the desired geometry of the flow passage: this could take the form of the orifice indicated in Figure 1(B) or a convergent-divergent nozzle.

The upper and lower pistons can be controlled, either individually or together, to generate the desired pressure and flow rate within the observation chamber. Piston velocities ranged from 0.01 to $10 \mathrm{~mm} / \mathrm{s}$, corresponding to volumetric flow rates ranging between 0.785 to $785 \mathrm{~mm}^{3} / \mathrm{s}$. Images of the bubble motion were recorded using the high speed camera.

The bubble dynamics and shape deformation were obtained from the bubble images by subsequent image analysis. The open source image analysis software FIJI (Schindelin et al., 2012) and Icy (de Chaumont et al., 2011) were used. Within Icy, the 'Active Contours' plugin (Dufour et al., 2011) was used to obtain data that enabled the bubble interface to be located. Data extracted from these tools was further processed using Origin ${ }^{\circledR}$ (OriginLab).

\footnotetext{
${ }^{1}$ Note to editor: supplementary material will be provided if this paper is accepted for publication.
} 


\section{Theory}

\subsection{Geometric theory for bubble deformation}

Figure 2 shows the basis of the theory. Figure 2(A) illustrates the flow pattern schematically: the flow field is assumed to be two-dimensional and radial, converging to and diverging away from the orifice marked ' $\mathrm{S}$ '. At a given radius, $r$, from $\mathrm{S}$ the radial velocity, $u_{r}$, is assumed to be constant. From continuity, the product of the radial velocity and the radial distance, $u_{r} r$, is constant.

\section{$<$ FIGURE 2>}

The second assumption concerns the behaviour of the bubble, shown in Figure 2(B) and Figure 2 (C). It is assumed that the bubble boundary, initially circular when viewed in cross section, moves as in the same manner as a region of the continuous phase marked with dye. This assumes that the effect of surface tension is small, corresponding to large capillary number, and that the effect of constant pressure within the bubble is negligible in comparison with the pressure variations within the continuous phase. This latter assumption is likely to be valid when the bubble diameter is small compared with the magnitude of the distances covered by the bubble when approaching and leaving the orifice.

The assumption that the bubble behaves in the same manner as an initially circular element of the continuous phase leads to a simple geometric theory to describe its shape at different points in the flow. A detailed derivation of this theory is given in the Appendix, but the principle is described here. Consider points A and B on the bubble surface, shown in Figure 2(A). The assumption of continuity, i.e. that the product $u_{r} r$ is constant leads to Equation $\mathrm{A} 2$ that relates the radius, $r$, to the time, $t$. This leads to Equation A4, which enables the relative movements of the front and back of the bubble to be predicted. Thus in Figure 2 (A), $A$ moves to $A^{\prime}$ and $B$ moves to $B^{\prime}$; likewise $P$ moves to $P^{\prime}$ and $Q$ moves to $Q^{\prime}$. The length $A^{\prime} B^{\prime}$ must be less than the length $A B$ because the back of the bubble always moves faster than the front, i.e. the velocity of $A^{\prime}$ is greater than that of $B^{\prime}$.

This velocity difference leads to the formation of 'crescent moon' bubbles shown in Figure 2(C): the successive positions of $A$ are shown by the asterisk; likewise the successive positions of $B$ are shown by the plus sign. The same analysis shows that for a bubble drawn towards the orifice, i.e. the upstream side of $\mathrm{S}$ in Figure 2 (A), the bubble must elongate in the direction of flow. This follows from the fact that the front of the bubble moves faster than the back. The equations that describe the shapes shown in Figure 2 (C) are given in the Appendix.

\subsection{Image analysis}

The 'Active Cells' plug-in (Delgado-Gonzalo et al., 2015) was used with Icy (de Chaumont et al., 2011) to carry out image analysis using the fast active contour method (Williams and Shah, 1992). The aim of the analysis was to obtain a set of co-ordinates defining the interface of the bubble in each of the 
sequence of images that were acquired. These data were then further processed using Origin ${ }^{\circledR}$ to give three measures of shape that are commonly used for discrete particles, roundness, elongation and convexity ratio (Mora and Kwan, 2000): these three measures are illustrated schematically in Figure 3.

\section{$<$ FIGURE 3>}

\section{Results}

\subsection{Bubble shapes: experimental}

Figure 4 is a sequence of photographs showing the appearance of an initially spherical bubble being driven through an orifice by an upward flow $\left(7.85 \mathrm{~mm}^{3} / \mathrm{s}\right)$. Although the apparatus was quasi twodimensional, the initial bubble was spherical and of diameter $745 \mu \mathrm{m}$ : this is of the same order of size as the orifice $(1.0 \mathrm{~mm})$. However, the bubble shapes observed exhibit forms similar to those predicted by the theory in Section 4. As the bubble is dragged upwards by the flow, it elongates in the direction of the flow, images (A) to (D). On passing through the orifice, the crescent shape emerges, images (F) to $(\mathrm{H})$.

\section{$<$ FIGURE 4>}

The remarkable variety of bubble shapes illustrated in Figure 4 share features observed by other authors. The 'egg-shaped' bubble in Figure 4(B) is similar to that shown by Dawson and co-workers (Dawson et al., 2015); the shape in Figure 4(D) is reminiscent of the 'long-tail' bubbles observed in a Hele-Shaw cell (Kopf-Sill and Homsy, 1988) and the 'crescent-moon' shapes in Figure 4(F) and (G) are similar to those resulting from the calculations by Khayat and co-workers (Khayat et al., 2000). Single cusps are visible in Figure 4(D), (E) and (F), with twin cusps being visible in Figure 4(H).

Figure 5 shows a sequence of images for a bubble passing twice through the orifice. Sequence (A), with downward flow, shows an initially elongated bubble passing down through an orifice. As it enters the orifice the elongation increases, as expected. Then then bubble emerging downwards shows the crescent shape, as expected. Sequence (B) shows the crescent-shaped bubble transform to an elongated bubble during up flow. But on emerging from the orifice, in the last frame on the right, the bubble again becomes crescent shaped.

\section{$<$ FIGURE 5>}

Again, a remarkable variety of bubble shapes are visible in Figure 5. The images in Figure 5(A) can be considered to derive from essentially the same experiment as illustrated in Figure 4, with single cusp 'long-tail' bubbles and twin cusp 'crescent-moon' bubbles being observed as before. The images in Figure 5(B) can be compared to those in observed in a channel containing multiple constrictions (Sauzade and Cubaud, 2018), notably the 'wedge-shaped' bubble in Figure 5(B-iii) and the 'plugshaped' bubble in Figure 5(B-iv). The influence of the initial bubble shape on the subsequent bubble 
deformation and breakup behaviour is also abundantly clear in Figure 5. Not only are there significant differences between the bubble shapes in Figure 5(B) when compared to Figure 5(A), but some breakup of the bubble is observed as it exits the orifice in the upstream direction, Figure 5(B-v) and Figure 5(Bvi).

Descriptions of droplet breakup for viscosity ratios as low as $10^{-2}$ (Stone, 1994) and critical capillary numbers for droplet breakup at viscosity ratios as low as $10^{-3}$ (Bentley and Leal, 1986) are given in the literature: the viscosity ratio in this experiment is $7 \times 10^{-6}$. Grace gives details of the breakup of initially spherical droplets in both shear and extensional flows with viscosity ratios as low as $10^{-6}$ for shear flow and $10^{-4}$ for extensional flows: critical capillary numbers of approximately 300 and 2, respectively, are required for breakup (Grace, 1982). The flow in Figure 5 contains both shear and extensional components, hence the critical capillary numbers quoted are likely to be the upper and lower bounds. The question of why droplet breakup is observed in Figure 5(B) and not in Figure 5(A), when one could reasonably argue that the capillary numbers are essentially the same in both experiments, can be answered by examining the difference in initial bubble shape. Stegeman and co-workers (Stegeman et al., 2008) build on the work by Grace by investigating the effect of initial droplet shape: they conclude that droplets that are initially stretched will breakup at capillary numbers lower than their spherical counterparts for the same viscosity ratio.

\subsection{Bubble shapes: theoretical}

Figure 6 shows theoretical predications for the shape of a two-dimensional bubble passing through an orifice: two stages can be identified.

(i) As the bubble moves up towards the orifice (red), it elongates, becoming taller and thinner.

(ii) When the bubble is wholly through the orifice (blue), the widening is dominant leading to the crescent-moon shape.

\section{$<$ FIGURE 6>}

\subsection{Bubble shapes: experiment and theory compared}

The shapes observed in experiments, Figure 4 and Figure 5, are qualitatively similar to those in Figure 6, despite the differences in the geometry between experiment and theory. Recall that (i) the experimental orifice shown in Figure 4 and Figure 5 has a finite height : width ratio whereas the theory treats the orifice as a combined point source and point sink and (ii) that the initial bubble in the experimental case, shown in Figure 4, is spherical whereas it would be cylindrical in the twodimensional theory. Motivated by the qualitative similarities, quantitative analysis of the bubble shape, both experimental and theoretical, was carried out. 
Figure 7 shows the three measures of bubble shape, roundness, elongation and convexity ratio, for an initially spherical bubble passing upwards through the orifice. The blue lines represent the experiment illustrated in Figure 4 and the red dashed lines predictions of bubble shape from the geometric theory. Also marked on this diagram are the approximate times of each of the images shown in Figure 4. The following observations can be made:

i. no single measure of bubble shape is sufficient to unambiguously describe the shape of the bubble;

ii. elongation ratio yields similar numerical values both upstream and downstream of the orifice despite strikingly different bubble shapes;

iii. a combination of roundness and convexity can be used to reasonably infer the shape changes the bubble undergoes: bubble stretching upstream of the orifice results in small values of roundness, but unity convexity ratio; within the orifice, the stretched bubbles return to a more spherical shape, shown by increasing roundness but still essentially unity convexity ratio; downstream of the orifice, as 'crescent-moons' form, both roundness and convexity ratio values decrease;

iv. the roundness and convexity ratio values computed for the geometric theory qualitatively capture some of the trends shown experimentally, whereas elongation values remain high due to the smaller radii of curvature resulting from the geometric theory.

\section{<FIGURE 8>}

Figure 8 shows shape measurements for an initially crescent-moon shaped bubble flowing upwards through an orifice. In a similar manner to Figure 7, blue lines represent the experiment illustrated in Figure 5(B), with the approximate times of each of the frames marked, and red dashed lines are predictions from the geometric theory. Similar observations can be made to those resulting from Figure 7 , notably (i) that a combination of roundness and convexity ratio appears to give good quantitative information concerning the change of bubble shape and (ii) that elongation ratio and roundness measurements derived from the geometric theory again qualitatively capture some of the trends observed experimentally. A further study by the same authors (Hallmark et al., 2019) has been carried out that compares predictions from computational fluid dynamics to images similar to those presented in this paper. 


\subsection{Displacement of bubble boundaries and capillary numbers}

$<$ FIGURE 9>

Figure 9 shows the displacements, $Y$, of different parts of the bubble as it passes through the orifice. Note that on the upstream side, the back of the bubble gets left behind the front due to elongation of the bubble. On the downstream side, the tips of the bubble get left behind as the crescent-shaped bubble gets wider.

\section{$<$ FIGURE 10>}

Figure 10 shows capillary numbers based on bubble velocity, $C a=\mu u_{b} / \gamma$, calculated as a function of time, for different parts of the same bubble. Velocity data for each part of the bubble, $u_{b}$, was obtained from displacement data, $Y$, by use of centrally-weighted finite differences accurate to fourth order viz

$$
u_{b}(t)=\frac{-Y(t+2 \Delta t)+8 Y(t+\Delta t)-8 Y(t-\Delta t)+Y(t-2 \Delta t)}{12 \Delta t}
$$

Here, $\Delta t$ represents the time between each location measurement: it is assumed to be constant. The velocity data derived from Equation (1) contained significant variation due to measurement noise, hence average capillary number data is also given in Figure 10. The average capillary number at time $t, \overline{C a}(t)$, is taken as

$$
\overline{C a}(t)=\frac{1}{5} \sum_{\tau=t-2 \Delta t}^{\tau=t+2 \Delta t} C a(\tau)
$$

The capillary number indicates the relative importance of viscous and surface tension forces. In all parts of the flow $C a>1$, with the orifice constricting having $30<C a<40$. Within the orifice constriction, the capillary number based on the velocity of the bubble centroid is typically larger than that calculated using the velocity of the front of the bubble. This is due to how the bubble shape is changing in this region: the highly extended bubble shown in Figure $4(\mathrm{C})$ is contracting to the form shown in Figure 4(D), hence the centroid of the bubble is moving more quickly than the front of the bubble. Upstream of the orifice, the reverse applies: the bubble is extending from that shown in Figure 4(A) to Figure 4(C). Consequently, the capillary number based on the velocity of the front of the bubble is higher than that calculated using the velocity of the bubble centroid. Downstream of the orifice, the bubble deformation is essentially normal to the flow direction: there is no significant difference in the velocities 
of the bubble centroid and of the front of the bubble. The capillary numbers in this region are, therefore, similar.

These measurements of capillary number may explain why the geometric theory shown in Figure 2 and Figure 6 works well. In all parts of the flow surface tension forces are dominated by viscous forces, hence the bubble can act as a 'passive marker' within the continuous phase and can deform readily to the crescent shapes.

\section{Conclusions}

A gas bubble in a liquid of large viscosity shows remarkable changes of shape as it is carried through an orifice by the liquid flow. With polybutene, whose viscosity is about 70,000 times that of water, the bubble elongates as it is moved towards the orifice. On emerging from the orifice, the bubble distorts into the shape of a crescent moon.

These changes of shape can be predicted by a simple kinematic model. The liquid flow converges as it approaches the orifice. The closer to the orifice, the higher the velocity thus a bubble approaching an orifice is elongated in the direction of the flow. After the bubble has passed through the orifice, it is in a region of diverging flow: the further from the orifice, the slower the velocity. Hence the back of the bubble is catching up with the front, giving the crescent moon shape, as observed.

These considerations led to a simple geometric theory to predict the bubble shape as it approaches and leaves the orifice. This theory gives reasonably good predictions of bubble shape. Differences between the theory and experiment are inevitable because:

i. the theory assumes two-dimensional geometry, so the initial bubble shape is assumed to be cylindrical;

ii. in the experiments, the liquid flowed between two parallel plates, so the velocity near the wall was small. The theory assumed two-dimensional flow, i.e. no velocity variation in the direction normal to the plane of observation.

Simple measures of bubble shape, namely roundness, elongation and convexity ratio, were calculated using data obtained from image analysis. Elongation alone gives ambiguous results but a combination of roundness and convexity ratio were found to be appropriate measures for the bubble shapes that were encountered.

\section{Acknowledgements}

The work was supported by EPSRC contract number EP/N00230X/1. The authors are grateful to P.J. Davidson, who initiated the project.

\section{Attributions}


Data accessibility. All datasets presented in this work would be available on request.

Author's contributions. The project was conceived by JFD, CHC and BH. The experimental work was done by CHC. The theory was developed by JFD. The text was written by JFD and BH. The literature survey was done by $\mathrm{BH}$. Diagrams were produced by JFD, $\mathrm{CHC}$ and $\mathrm{BH}$. Data analysis was done by $\mathrm{CHC}$ and $\mathrm{BH}$.

Competing interests. The authors have no competing interests. 


\section{Nomenclature}

\section{Roman letters}

$\begin{array}{llll}c & - & \text { Integration constant for Equation (4) } & \left(\mathrm{m}^{2}\right) \\ k & - & \text { Normalised volumetric flow per unit width, } Q / \pi & \left(\mathrm{m}^{2} / \mathrm{s}\right) \\ Q & - & \text { Volumetric flow rate per unit width } & \left(\mathrm{m}^{2} / \mathrm{s}\right) \\ R_{0} & - & \text { Initial radius of spherical bubble } & (\mathrm{m}) \\ r & - & \text { Radius from orifice } \mathrm{S} & (\mathrm{m}) \\ \Delta t & - & \text { Time between measurements } \\ t & - & \text { Time } & (\mathrm{s}) \\ u_{r} & - & \text { Radial velocity } & (\mathrm{s}) \\ u & - & \text { Velocity } & (\mathrm{m} / \mathrm{s}) \\ u_{b} & - & \text { Bubble velocity } & (\mathrm{m} / \mathrm{s}) \\ Y & - & \text { Bubble displacement } & (\mathrm{m} / \mathrm{s}) \\ & & (\mathrm{m})\end{array}$

\section{Greek letters}

$\begin{array}{llll}\gamma & - & \text { Surface tension } & (\mathrm{N} / \mathrm{m}) \\ \mu & - & \text { Viscosity } & (\mathrm{Pa} \mathrm{s}) \\ \tau & - & \text { Time used for averaging } & \text { (s) }\end{array}$

\section{Non-dimensional groups}

$\begin{array}{lll}\mathrm{Ca} & - & \text { Capillary number, } \mu u_{b} / \gamma \\ \overline{\mathrm{Ca}} & - & \text { Average capillary number }\end{array}$

$(-)$ 


\section{References}

Amran, Y.H.M., Farzadnia, N., Abang Ali, A.A., 2015. Properties and applications of foamed concrete; a review. Constr. Build. Mater. 101, 990-1005.

Bentley, B.J., Leal, L.G., 1986. An experimental investigation of drop deformation and breakup in steady, two-dimensional linear flows. J. Fluid Mech. 167, 241.

Collishaw, P.G., Evans, J.R.G., 1994. An assessment of expressions for the apparent thermal conductivity of cellular materials. J. Mater. Sci. 29, 2261-2273.

Dawson, G., Häner, E., Juel, A., 2015. Extreme Deformation of Capsules and Bubbles Flowing through a Localised Constriction. Procedia IUTAM 16, 22-32.

de Chaumont, F., Dallongeville, S., Olivo-Marin, J.-C., 2011. ICY: A new open-source community image processing software, in: 2011 IEEE International Symposium on Biomedical Imaging: From Nano to Macro. IEEE, pp. 234-237.

Delgado-Gonzalo, R., Uhlmann, V., Schmitter, D., Unser, M., 2015. Snakes on a Plane: A perfect snap for bioimage analysis. IEEE Signal Process. Mag. 32, 41-48.

Drenckhan, W., Hutzler, S., 2015. Structure and energy of liquid foams. Adv. Colloid Interface Sci. 224, $1-16$.

Dufour, A., Thibeaux, R., Labruyere, E., Guillen, N., Olivo-Marin, J.-C., 2011. 3-D Active Meshes: Fast Discrete Deformable Models for Cell Tracking in 3-D Time-Lapse Microscopy. IEEE Trans. Image Process. 20, 1925-1937.

Eaves, D., 2004. Handbook of Polymer Foams, Handbook of Polymer Foams. iSmithers Rapra Publishing, Shrewsbury, UK.

Grace, H.P., 1982. Dispersion phenomena in high viscosity immiscible fluid systems and application of static mixers as dispersion devices in such systems. Chem. Eng. Commun. 14, 225-277.

Hallmark, B., Chen, C.-H., Davidson, J.F., 2019. Experimental and simulation studies of the shape and motion of an air bubble contained in a highly viscous liquid flowing through an orifice constriction. Chem. Eng. Sci. DOI: 10.1016/J.CES.2019.04.043.

Hill, C., Eastoe, J., 2017. Foams: From nature to industry. Adv. Colloid Interface Sci. 247, 496-513.

Hinch, E.J., Acrivos, D.A., 1980. Long slender drops in a simple shear flow. J. Fluid Mech 98, 305328.

Kameda, M., Katsumata, T., Ichihara, M., 2008. Deformation of bubbles in a highly viscous pipe flow. Fluid Dyn. Res. 40, 576-584. 
Khayat, R.E., Luciani, A., Utracki, L.A., Godbille, F., Picot, J., 2000. Influence of shear and elongation on drop deformation in convergent-divergent flows. Int. J. Multiph. Flow 26, 17-44.

Kopf-Sill, A.R., Homsy, G.M., 1988. Bubble motion in a Hele-Shaw cell. Phys. Fluids 31, 18-26.

Lewandowski, F.Y., Dupuis, D., 1994. Dynamic measurements of surface tension of solutions of polyisobutylene in mixtures of polybutene oil and Decalin. J. Nonnewton. Fluid Mech. 52, 233248.

Mackley, M.R., Marshall, R.T.J., Smeulders, J.B.A.F., 1995. The multipass rheometer. J. Rheol. (N. Y. N. Y). 39, 1293-1309.

Mader, H.M., Llewellin, E.W., Mueller, S.P., 2013. The rheology of two-phase magmas: A review and analysis. J. Volcanol. Geotherm. Res. 257, 135-158.

Mezzenga, R., Schurtenberger, P., Burbidge, A., Michel, M., 2005. Understanding foods as soft materials. Nat. Mater. 4, 729-740.

Mora, C.F., Kwan, A.K.H., 2000. Sphericity, shape factor, and convexity measurement of coarse aggregate for concrete using digital image processing. Cem. Concr. Res. 30, 351-358.

Narsimhan, G., Xiang, N., 2018. Role of Proteins on Formation, Drainage, and Stability of Liquid Food Foams. Annu. Rev. Food Sci. Technol. 9, 45-63.

Ostwald, W., 1897. Studien uber die Bildung und Umwandlung fester Koerper. Zeitschrift fur Phys. Chemie. 22, 189-330.

Pozrikidis, C., 1998. Numerical studies of cusp formation at fluid interfaces in Stokes flow. J. Fluid Mech. 357, 29-57.

Prince, M.J., Blanch, H.W., 1990. Bubble coalescence and break-up in air-sparged bubble columns. AIChE J. 36, 1485-1499.

Rallison, J.M., 1984. The Deformation of Small Viscous Drops and Bubbles in Shear Flows. Annu. Rev. Fluid Mech. 16, 45-66.

Richardson, J.F., Zaki, W.N., 1954. The sedimentation of a suspension of uniform spheres under conditions of viscous flow. Chem. Eng. Sci. 3, 65-73.

Richardson, S., 1973. Two-dimensional bubbles in slow viscous flows. Part 2. J. Fluid Mech. 58, 115.

Rust, A.C., Manga, M., 2002. Bubble Shapes and Orientations in Low Re Simple Shear Flow. J. Colloid Interface Sci. 249, 476-480.

Rust, A.C., Manga, M., Cashman, K.V., 2003. Determining flow type, shear rate and shear stress in 
magmas from bubble shapes and orientations. J. Volcanol. Geotherm. Res. 122, 111-132.

Sauzade, M., Cubaud, T., 2018. Bubble deformations and segmented flows in corrugated microchannels at large capillary numbers. Phys. Rev. Fluids 3, 034202.

Schindelin, J., Arganda-Carreras, I., Frise, E., Kaynig, V., Longair, M., Pietzsch, T., Preibisch, S., Rueden, C., Saalfeld, S., Schmid, B., Tinevez, J.-Y., White, D.J., Hartenstein, V., Eliceiri, K., Tomancak, P., Cardona, A., 2012. Fiji: an open-source platform for biological-image analysis. Nat. Methods 9, 676-682.

Shikhmurzaev, Y.D., 1998. On cusped interfaces. J. Fluid Mech. 359, 313-328.

Siegel, M., 2000. Cusp formation for time-evolving bubbles in two-dimensional Stokes flow. J. Fluid Mech. 412, S002211200000834X.

Stegeman, Y.W., Van De Vosse, F.N., Meijer, H.E.H., 2008. On the Applicability of the Grace Curve in Practical Mixing Operations. Can. J. Chem. Eng. 80, 1-6.

Stone, H.A., 1994. Dynamics of Drop Deformation and Breakup in Viscous Fluids. Annu. Rev. Fluid Mech. 26, 65-102.

Torres, M.D., Hallmark, B., Wilson, D.I., 2015. Effect of bubble volume fraction on the shear and extensional rheology of bubbly liquids based on guar gum (a Giesekus fluid) as continuous phase. J. Food Eng. 146.

Voorhees, P.W., 1985. The Theory of Ostwald Ripening. J. Stat. Phys. 38, 231-252.

Wallis, G.B., 1974. The terminal speed of single drops or bubbles in an infinite medium. Int. J. Multiph. Flow 1, 491-511.

Wang, J., 2009. Rheology of Foaming Polymers and Its Influence on Microcellular Processing. University of Toronto.

Williams, D.J., Shah, M., 1992. A Fast algorithm for active contours and curvature estimation. CVGIP Image Underst. 55, 14-26. 


\section{Appendix}

Consider the plate PP containing a small hole, S, that is shown in Figure 2(A). When highly viscous liquid is forced through $\mathrm{S}$, we assume that the flow is radially inwards on the upstream side, and radially outwards on the downstream side. In the experiments described in this paper, the liquid viscosity was $70 \mathrm{~Pa}$ s. The forces arising on the bubble from viscosity will therefore be significantly larger than those resulting from surface tension.

The prediction of bubble shapes in this analysis assumes that the motion of the bubble periphery is the same as for a circular slug of dye put into the liquid. The subsequent motion of the dye in the liquid is assumed to be the same as that of the bubble surface. This assumption is likely to be true for very small bubbles with zero surface tension.

The equations derived below are for 'two-dimensional' motion, i.e. all velocities are in the plane of the diagrams. In Figure A 1 , we follow a bubble, initially of radius $R_{0}$, in radial flow away from a point source, S. Now consider the velocities. The volumetric flow rate for a unit thickness is $Q \mathrm{~m}^{2} / \mathrm{s}$, hence $Q=\pi r u_{r}, r$ being the radius measured from $\mathrm{S}$ and $u_{r}$ is the radial velocity at radius $r$. Thus

$$
u_{r}=\frac{k}{r}=\frac{d r}{d t}
$$

where $k=Q / \pi$. Integrating Equation (3) with respect to $r$ gives

$$
r^{2}=2 k t+c
$$

where $c$ is a constant of integration. Equation (4) applies to all elements, so the relationship between initial and final positions are governed by this expression. Thus, for an element starting at position B,

$$
r_{3}^{2}-r_{B}^{2}=2 k t\left(t_{3}-t_{B}\right)
$$

where $r_{3}$ is the final position of element $\mathrm{B}$. The times $t_{B}$ and $t_{3}$ refer to points $\mathrm{B}$ and 3 . The time interval, $\left(t_{3}-t_{B}\right)$ is the same for all elements, so

$$
r_{3}^{2}-r_{B}^{2}=r_{2}^{2}-r_{F}^{2}=r_{1}^{2}-r_{0}^{2}
$$

The term $r_{1}^{2}-r_{0}^{2}$, relating the edge of the bubble, can be chosen to give a series of positions for the bubble so 


$$
\begin{aligned}
& r_{2}^{2}=r_{F}^{2}+r_{1}^{2}-r_{0}^{2} \\
& r_{3}^{2}=r_{B}^{2}+r_{1}^{2}-r_{0}^{2}
\end{aligned}
$$

Equations (7) determines the shape of the bubble. For large values of $r_{0}$, the term $r_{1}^{2}-r_{0}^{2}$ will dominate, so $r_{3}$ will not be much less than $r_{2}$, giving the crescent shapes shown in the photographs. The crescent shape arises from the fact that the back of the bubble moves faster than the front, because $u_{r}=k / r$ from Equation (3).

Following the analysis above, for a bubble passing through an orifice $S$, the bubble shape downstream can be predicted from the movements shown in Figure A 2. Point T moves to R, point B moves to $r_{3}$, $r_{0}$ moves to $r_{1}, \mathrm{C}$ moves to $\mathrm{D}$ and $\mathrm{F}$ moves to $r_{2}$. From the fact that all elements have the same time lapse for the bubble, the above argument gives

$$
\begin{aligned}
& r_{1}^{2}=r_{C}^{2}+r_{D}^{2}-r_{0}^{2} \\
& r_{2}^{2}=r_{C}^{2}+r_{D}^{2}-r_{F}^{2} \\
& r_{3}^{2}=r_{C}^{2}+r_{D}^{2}-r_{B}^{2} \\
& r_{R}^{2}=r_{C}^{2}+r_{D}^{2}-r_{T}^{2}
\end{aligned}
$$

The procedure is to choose a succession of values of $r_{D}$; for each $r_{D}$ Equations (8) will give $r_{1}, r_{2}, r_{3}$ and $r_{R}$. As before, if $r_{D}$ is large, $r_{3}$ will be only a little less than $r_{2}$, giving a crescent-shaped bubble.

Consider Figure A 3. Here, the bubble is in converging flow, but no part of the it reaches the orifice: thus the bottom of the bubble, point $\mathrm{D}$, does not reach $\mathrm{S}$. Note that the converging flow elongates the bubble, i.e. its height becomes greater than its width. Because of the converging flow, the bottom of the bubble travels faster than the top, hence explaining the elongating effect. This shows up in the 'pointed' form of the bottom of the bubble, as seen in the experiments and in Figure 6.

It is possible, though not too straightforward, to show that as a bubble distorts in converging or diverging flow, its area is constant. This is as it should be, for the above analysis simply follows the motion of liquid elements as if the gas bubble was not there. The above analysis can also be readily adapted to the case where the bubble is part way through the orifice; the top part of the bubble is in converging flow, as in Figure A 3. The lower part of the bubble is in diverging flow, as shown in Figure A 1, this resulting in a crescent-shaped form on the downstream side of the orifice. 


\section{List of figures}

Figure 1. (A) Schematic diagram of the MPR - the observation region is shown in the dotted black box. (B) schematic diagram, not to scale, of the orifice plate showing key dimensions.

Figure 2. Geometric method of predicting bubble shapes from an assumed flow pattern (A). Radial liquid flow to and from $\mathrm{S}$ is assumed. In (B), point $\boldsymbol{A}$ moves to $\boldsymbol{A}^{\prime}$ along the intermediate positions shown, * and + , in (C); likewise $\boldsymbol{B}, \boldsymbol{P}$ and $\boldsymbol{Q}$. The crescent shapes in (C) were formed using this principal.

Figure 3. Schematic diagram illustrating (A) roundness measurement, $\boldsymbol{R}_{\mathbf{1}} / \boldsymbol{R}_{\mathbf{2}}$; (B) elongation measurement, $\boldsymbol{b} / \boldsymbol{a} ;(\mathrm{C})$ convexity ratio measurement $\boldsymbol{A}_{\mathbf{1}} / \boldsymbol{A}_{\mathbf{2}}$.

Figure 4. Sequence of images, (A) to (H) showing an initially spherical bubble passing upwards through an orifice. Initial bubble diameter $\sim 745 \boldsymbol{\mu m}$

Figure 5. Sequence of images, (A-i) to (A-vi), showing an initially spherical bubble (out of view) passing downwards through an orifice forming crescent-shaped bubbles. Second sequence of images, (B-i) to (B-vi), showing the crescent-shaped bubble illustrated in (A-vi) passing upwards through the same orifice.

Figure 6. Prediction of bubble shapes from the geometric theory shown schematically in Figure 2. An initially elliptical bubble, labelled, contracts as it approaches an orifice, $\mathrm{S}$, and then expands to become crescent-shaped as it passes away from the orifice.

Figure 7. Measurements of (A) roundness, (B) elongation and (C) convexity as a function of time for a bubble passing upwards through an orifice (shown by vertical grey bar). Continuous lines represent experimental data taken from an initially spherical bubble, chain-dashed lines represent data taken from the geometric theory for a bubble initially circular in section.

Figure 8. Measurements of (A) roundness, (B) elongation and (C) convexity as a function of time for a bubble passing upwards through an orifice (shown by vertical grey bar). Continuous lines represent experimental data taken from an initially crescent-shaped bubble, chain-dashed lines represent data taken from the geometric theory for a bubble with a crescent-shaped cross-section.

Figure 9. Displacement of different parts of an initially spherical bubble as a function of time as the bubble flows upwards through an orifice (shown in grey). Volumetric flowrate of continuous phase 7.85 $\mathrm{mm} 3 / \mathrm{s}$.

Figure 10. Capillary number, $\mathbf{C a}=\boldsymbol{\mu} \boldsymbol{u}_{\boldsymbol{b}} / \boldsymbol{\gamma}$, as a function of time for different locations of an initially spherical bubble passing upwards through an orifice. Definitions of 'front' and 'centroid' shown in inset for a crescent-shaped bubble.

Figure A 1. Movements of elements in radial flow from a point source S. $\boldsymbol{r}_{\mathbf{2}}$ and $\boldsymbol{r} \mathbf{3}$ to scale from Equation (7). Movements shown are not to scale.

Figure A 2. Bubble trajectory as it passes through an orifice, S.

Figure A 3. Bubble approaching an orifice. 


\section{Figures}

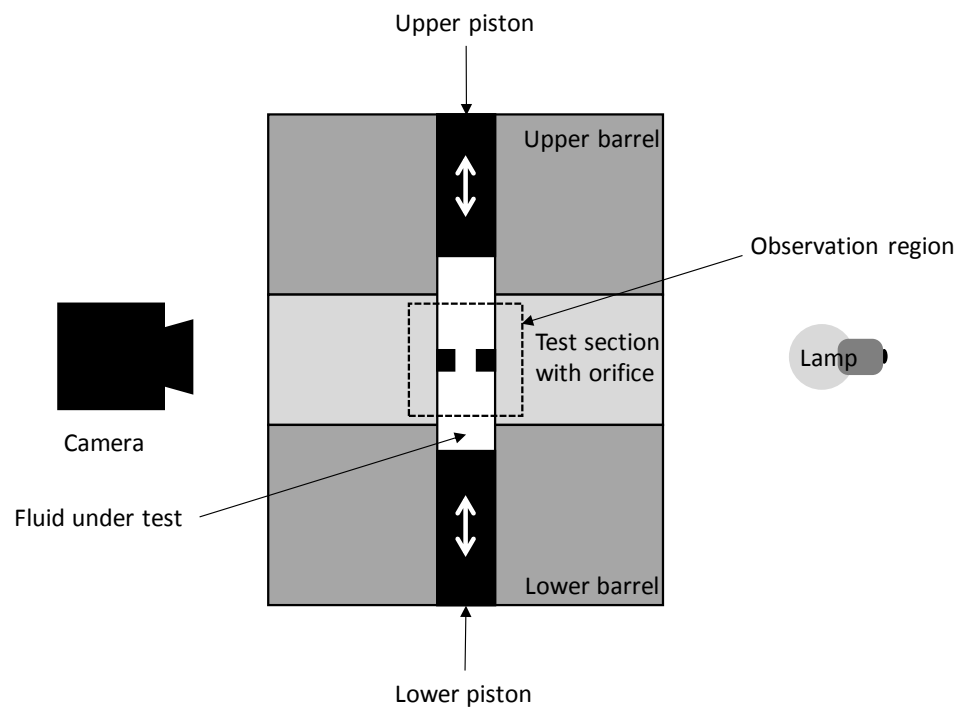

(A)

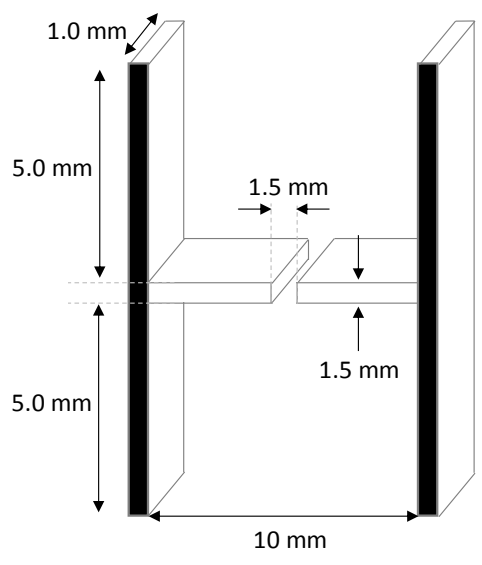

(B)

Figure 1. (A) Schematic diagram of the MPR - the observation region is shown in the dotted black box. (B) schematic diagram, not to scale, of the orifice plate showing key dimensions.

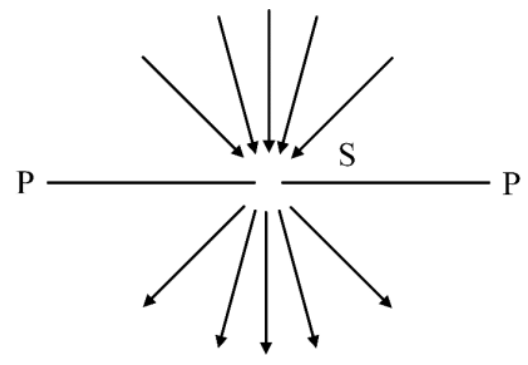

(A)

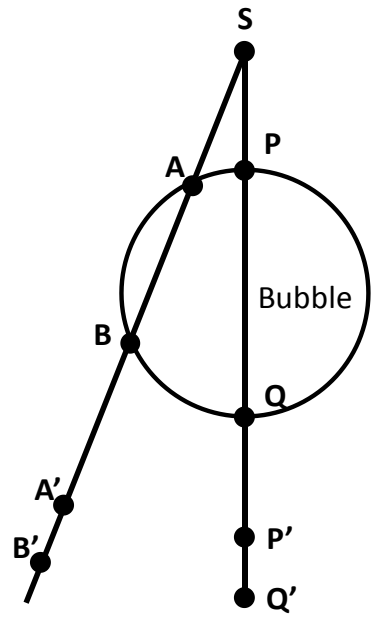

(B)

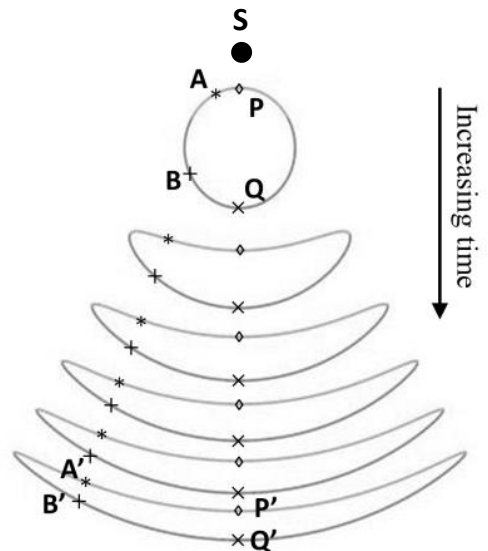

(C)

Figure 2. Geometric method of predicting bubble shapes from an assumed flow pattern (A). Radial liquid flow to and from $S$ is assumed. In (B), point $A$ moves to $A^{\prime}$ along the intermediate positions shown, * and +, in (C); likewise $B, P$ and $Q$. The crescent shapes in $(C)$ were formed using this principal. 


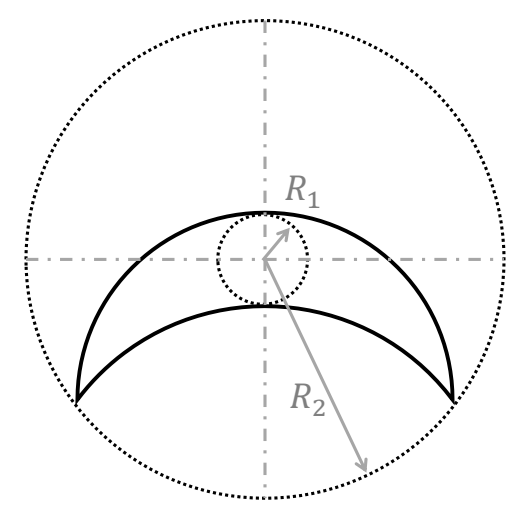

(A)

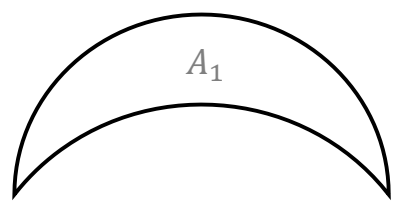

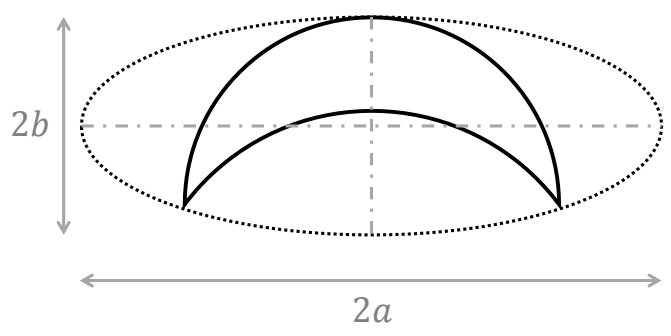

(B)

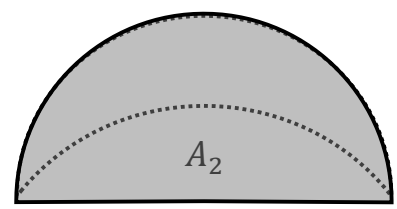

(C)

Figure 3. Schematic diagram illustrating (A) roundness measurement, $R_{1} / R_{2}$; (B) elongation measurement, $b / a ;(C)$ convexity ratio measurement $A_{1} / A_{2}$.

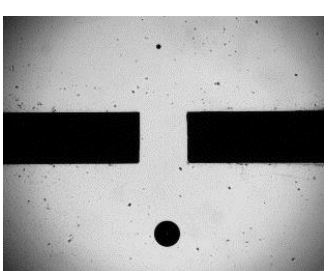

(A)

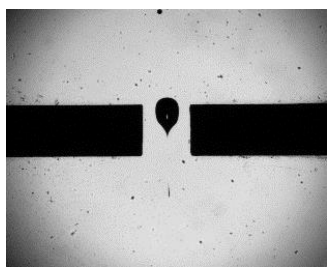

(E)

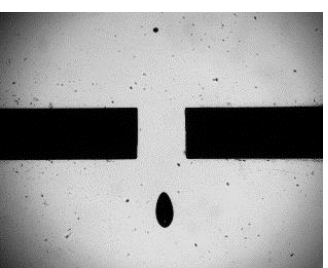

(B)

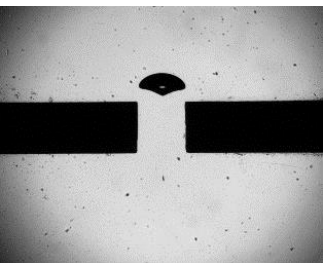

(F)

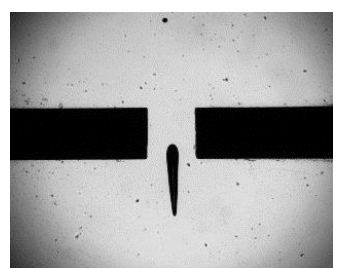

(C)

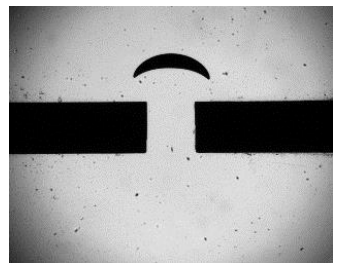

(G)

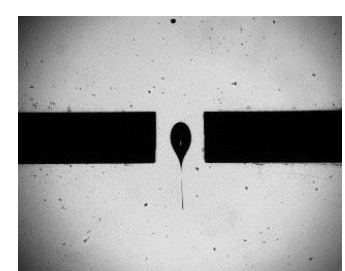

(D)

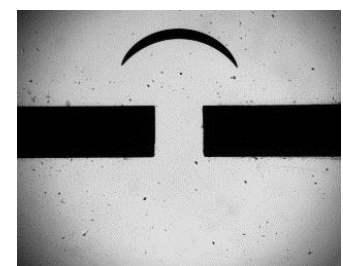

(H)

$3.0 \mathrm{~mm}$

Figure 4. Sequence of images, (A) to (H) showing an initially spherical bubble passing upwards through an orifice. Initial bubble diameter $\sim 745 \mu \mathrm{m}$, volumetric flow of continuous phase $7.85 \mathrm{~mm}^{3} / \mathrm{s}$. 


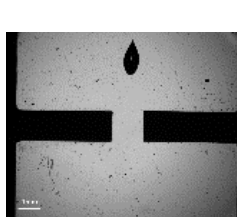

(A-i)

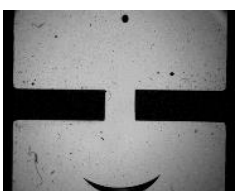

(B-i)

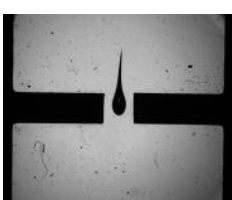

(A-ii)

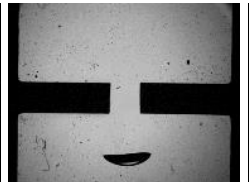

(B-ii)

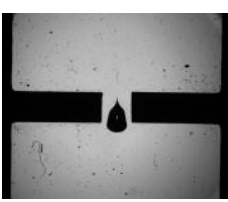

(A-iii)

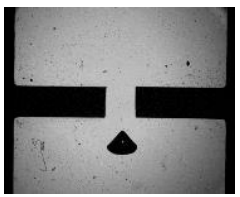

(B-iii)

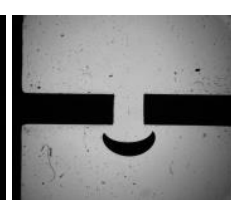

(A-iv)

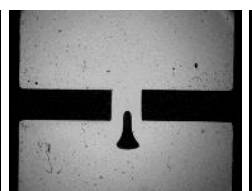

(B-iv)

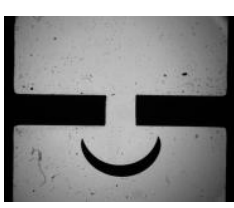

(A-v)

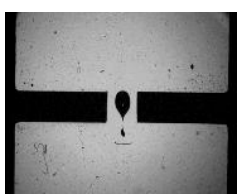

(B-v)

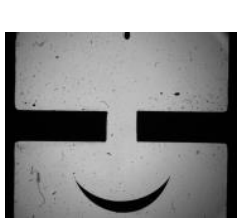

(A-vi)

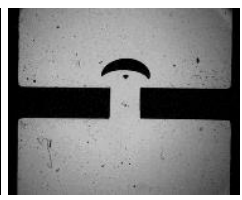

(B-vi)

$3.0 \mathrm{~mm}$

Figure 5. Sequence of images, (A-i) to (A-vi), showing an initially spherical bubble (out of view) passing downwards through an orifice forming crescent-shaped bubbles. Second sequence of images, (B-i) to (Bvi), showing the crescent-shaped bubble illustrated in (A-vi) passing upwards through the same orifice.

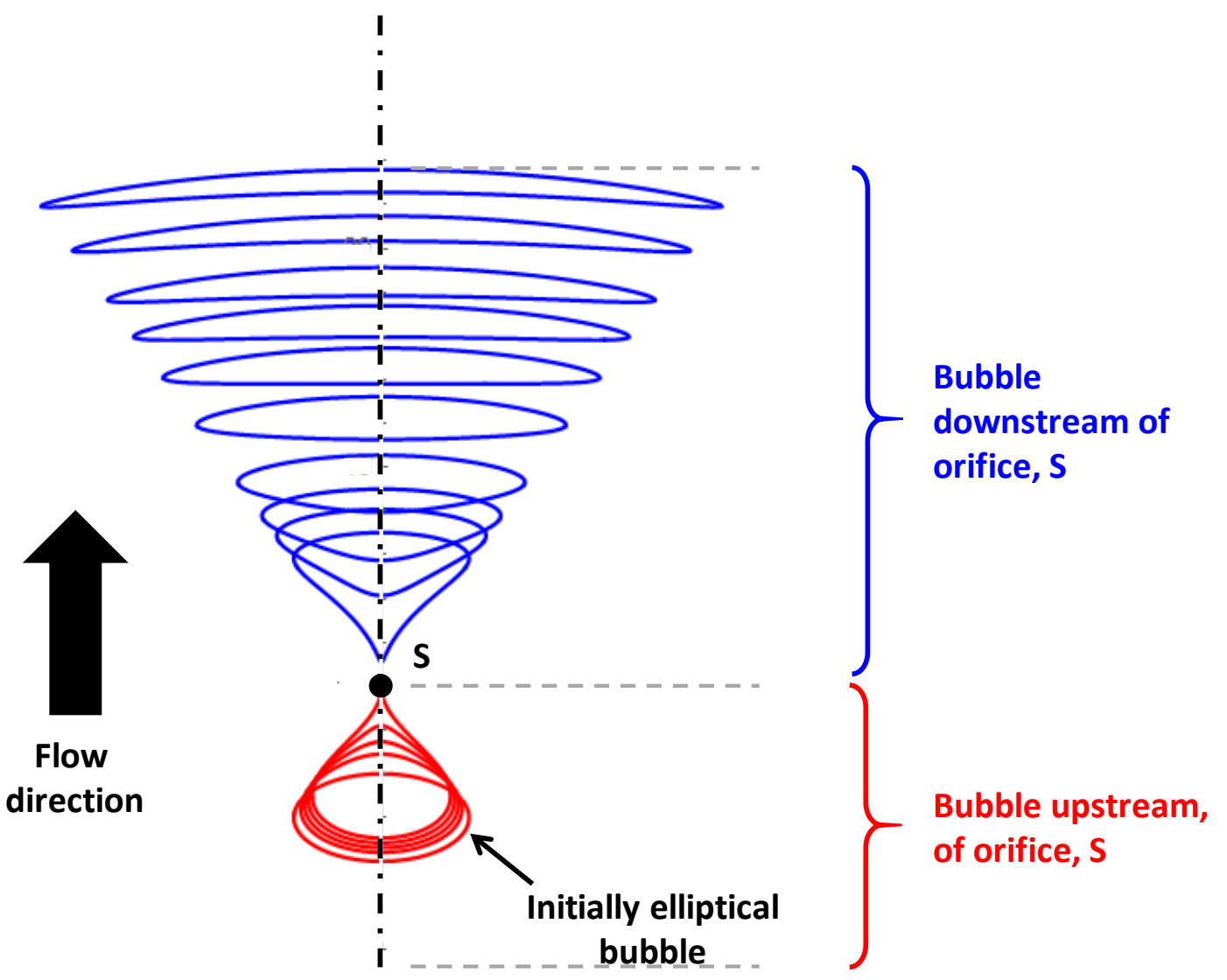

Figure 6. Prediction of bubble shapes from the geometric theory shown schematically in Figure 2. An initially elliptical bubble, labelled, contracts as it approaches an orifice, $S$, and then expands to become crescent-shaped as it passes away from the orifice. 


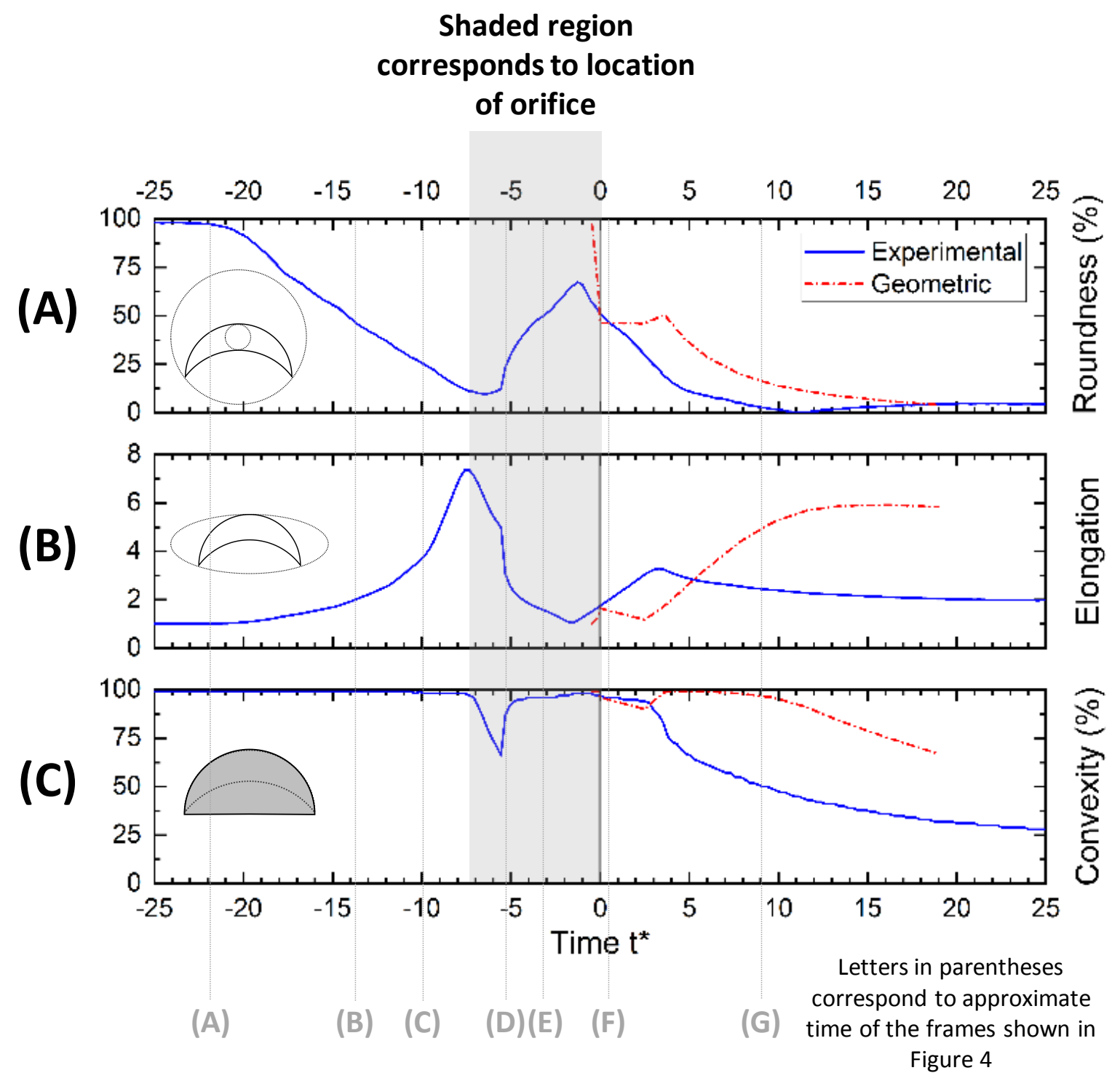

Figure 7. Measurements of $(\mathrm{A})$ roundness, $(\mathrm{B})$ elongation and $(\mathrm{C})$ convexity as a function of time for a bubble passing upwards through an orifice (shown by vertical grey bar). Continuous lines represent experimental data taken from an initially spherical bubble, chain-dashed lines represent data taken from the geometric theory for a bubble initially circular in section. 


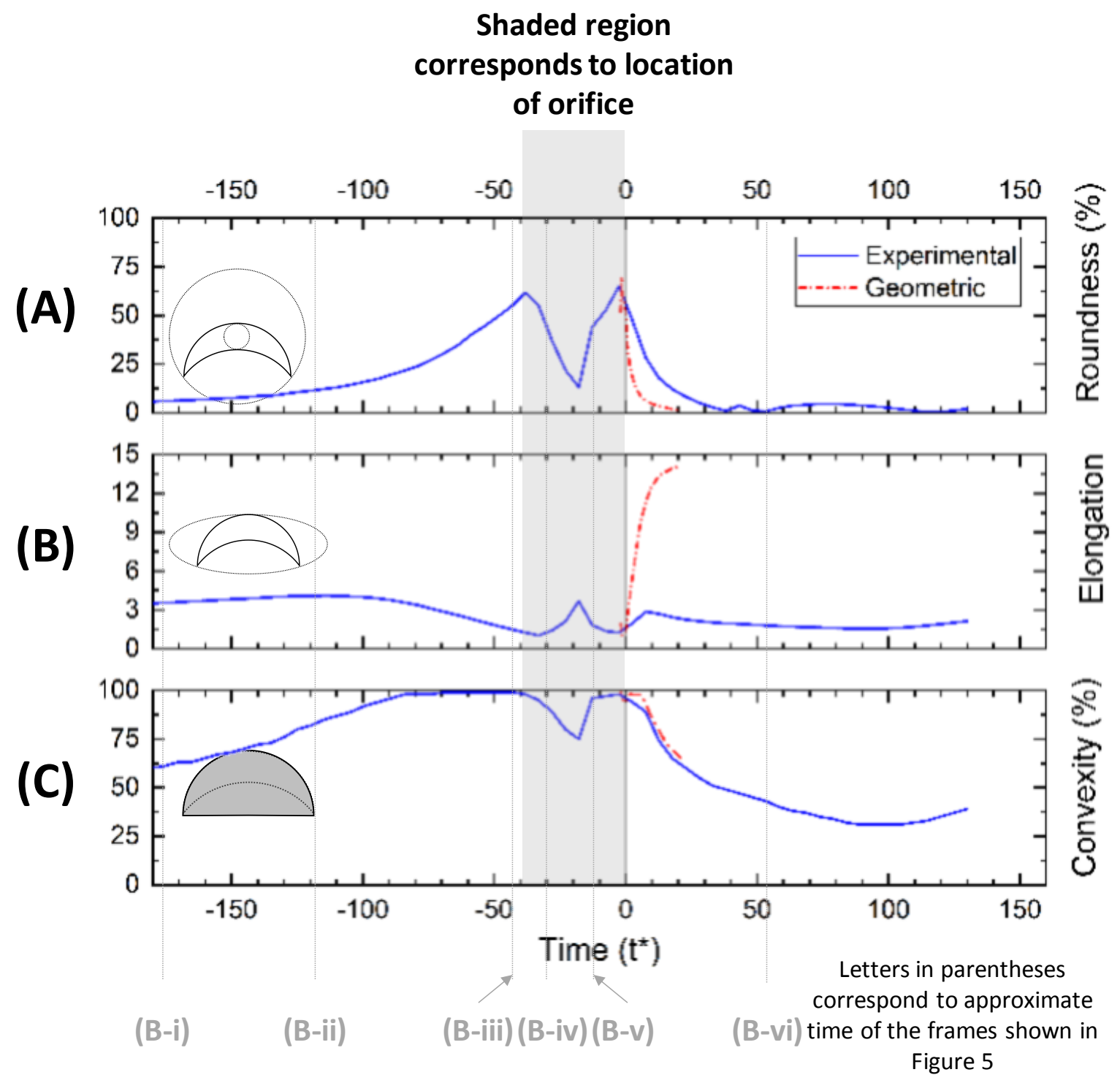

Figure 8. Measurements of $(\mathrm{A})$ roundness, $(\mathrm{B})$ elongation and $(\mathrm{C})$ convexity as a function of time for a bubble passing upwards through an orifice (shown by vertical grey bar). Continuous lines represent experimental data taken from an initially crescent-shaped bubble, chain-dashed lines represent data taken from the geometric theory for a bubble with a crescent-shaped cross-section. 


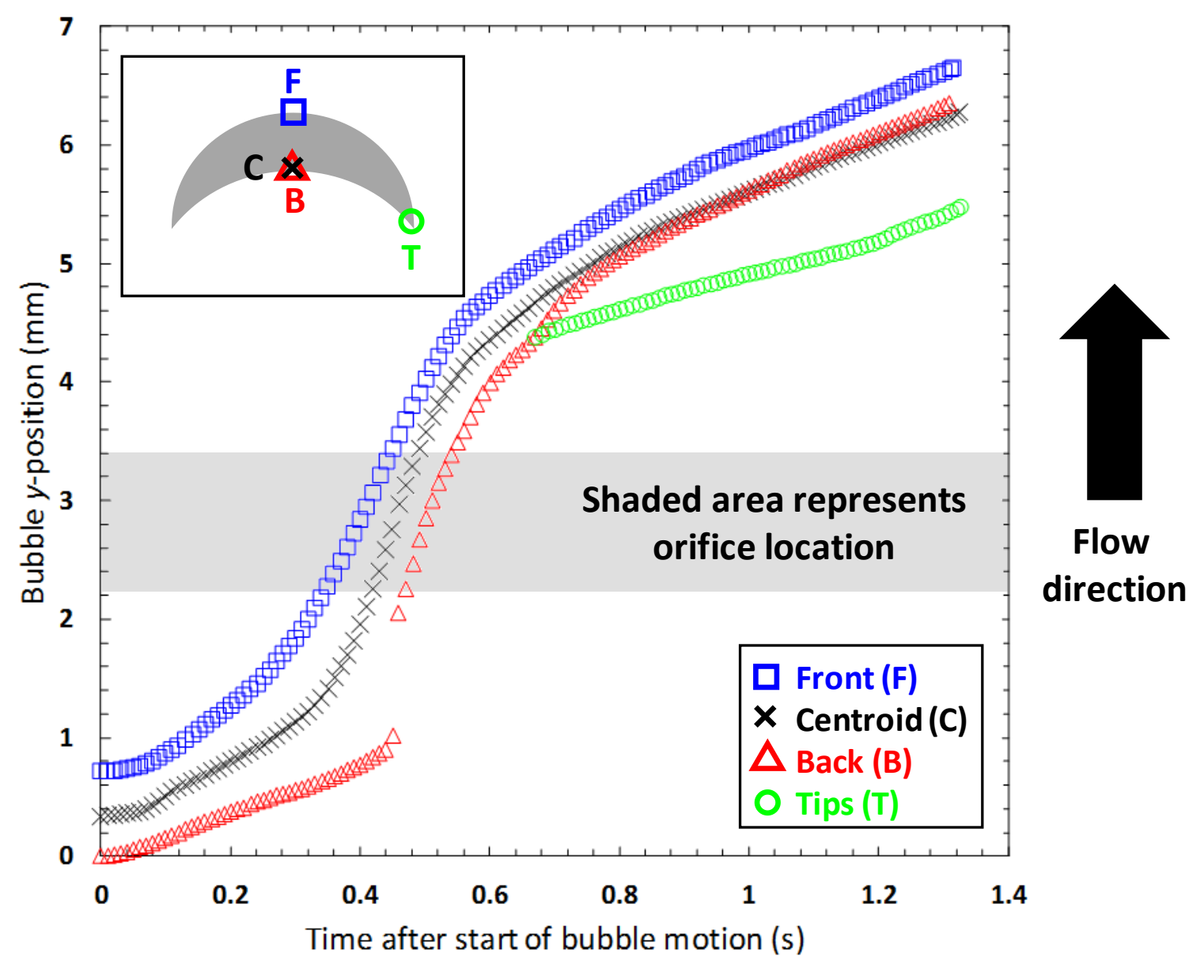

Figure 9. Displacement of different parts of an initially spherical bubble as a function of time as the bubble flows upwards through an orifice (shown in grey). Volumetric flowrate of continuous phase $7.85 \mathrm{~mm}^{3} / \mathrm{s}$. 


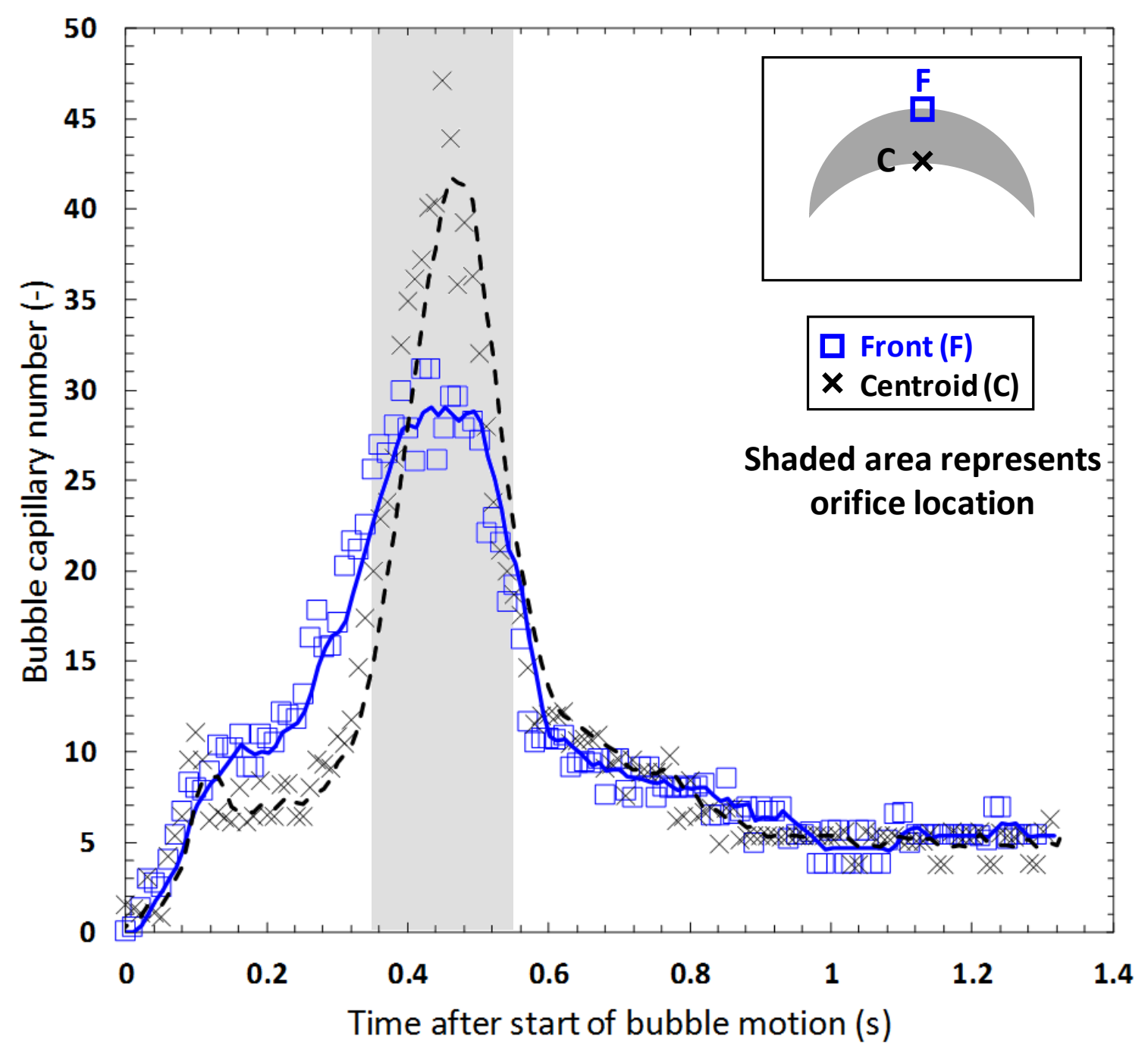

Figure 10. Capillary number, $\mathrm{Ca}=\mu \boldsymbol{u}_{b} / \gamma$, as a function of time for different locations of an initially spherical bubble passing upwards through an orifice. Definitions of 'front' and 'centroid' shown in inset for a crescent-shaped bubble. Individual symbols represent instantaneous Capillary number data; lines represent the average capillary number, Equation (2). 


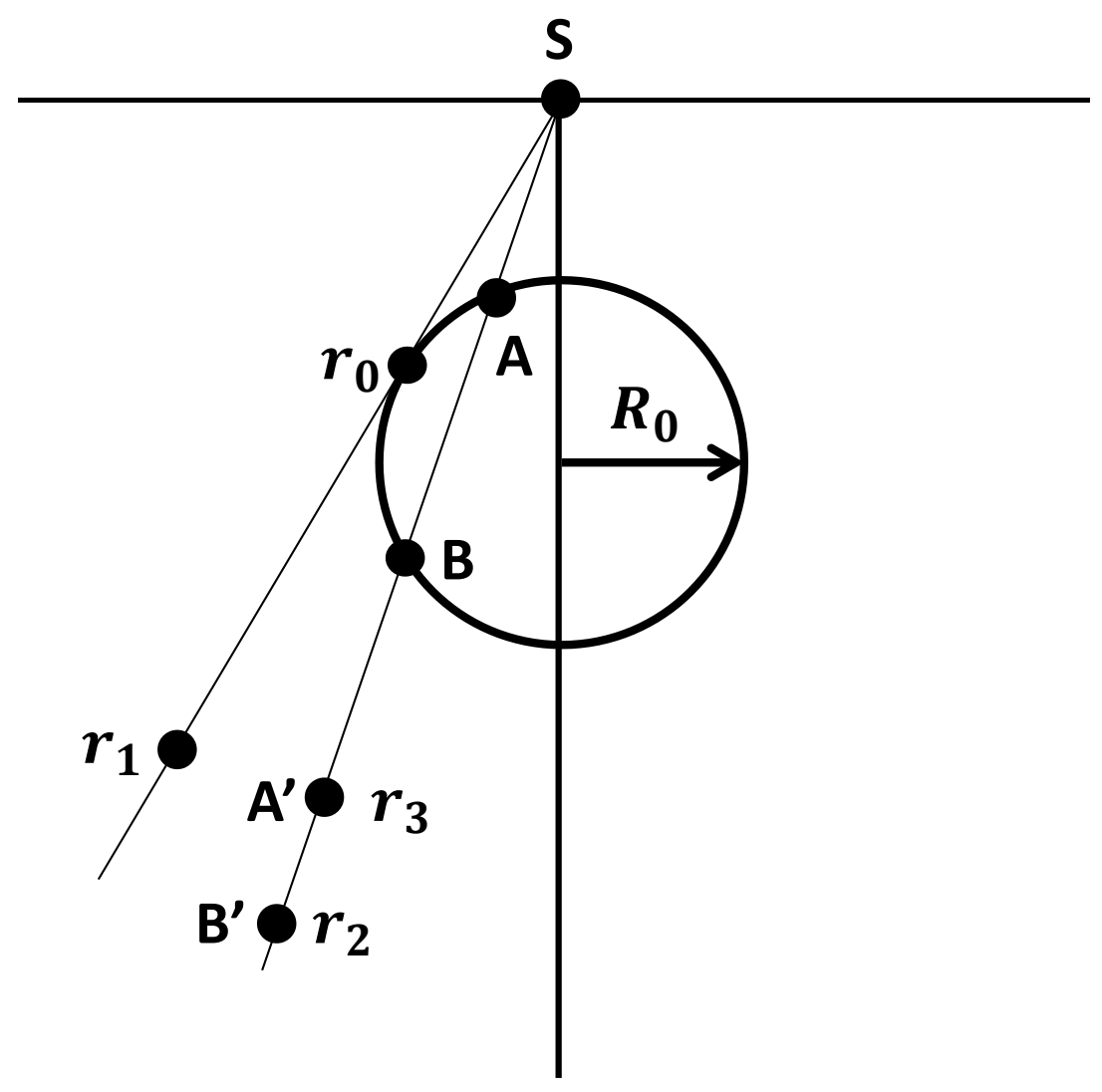

Figure A 1. Movements of elements in radial flow from a point source $S . \boldsymbol{r}_{2}$ and $\boldsymbol{r}_{3}$ to scale from Equation (7). Movements shown are not to scale. 


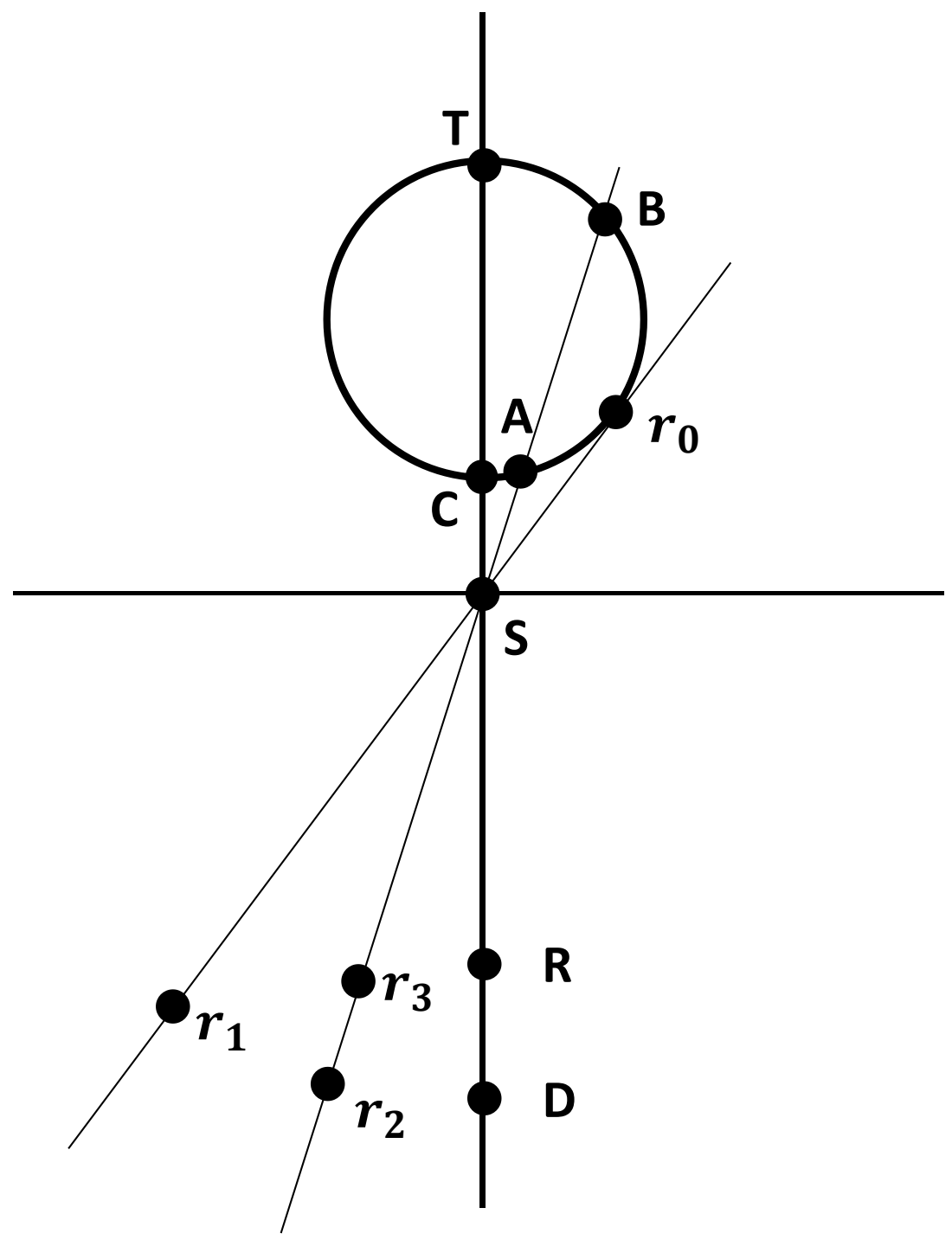

Figure A 2. Bubble trajectory as it passes through an orifice, $S$. 


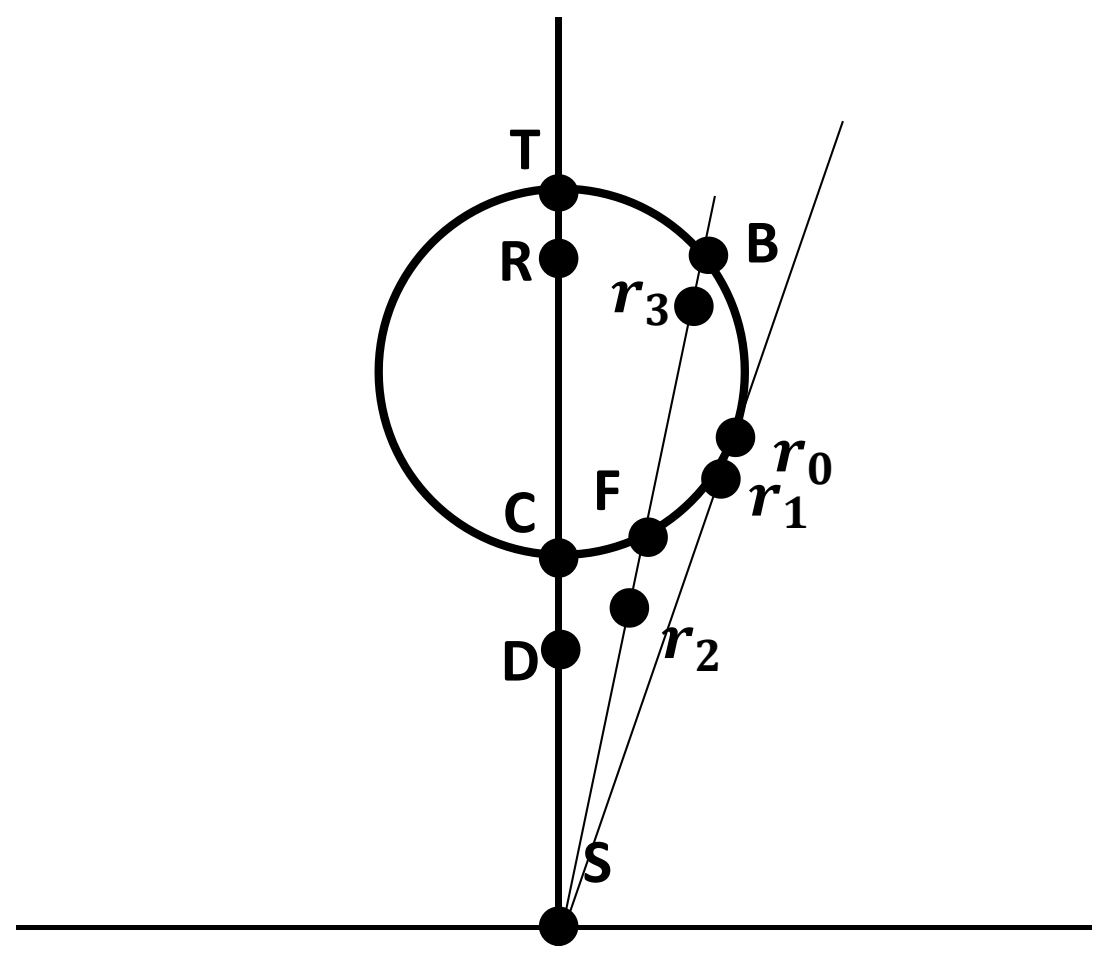

Figure A 3. Bubble approaching an orifice. 